\title{
Chaos synchronization of fractional-order discrete-time systems with different dimensions using two scaling matrices
}

https://doi.org/10.1515/phys-2019-0099

Received May 06, 2018; accepted Jul 30, 2019

Abstract: In this paper, we study the synchronization of fractional-order discrete-time chaotic systems by means of two scaling matrices $\Theta$ and $\Phi$. The considered synchronization scheme can be tailored to encompass several types of classical synchronization types. We proposed two nonlinear control strategies for the $\Theta-\Phi$ synchronization of an $m$-dimensional drive system and an $n$-dimensional response system, whereby the synchronization dimension $d=m$ and $d=n$, respectively. Numerical examples are presented to test the findings of the study.

Keywords: Chaotic maps, discrete-time fractional calculus, synchronization, different dimensions, linear systems, stability

PACS: 34A08, 34H10, 34D06

\section{Introduction}

Discrete-time chaotic systems have attracted a low of attention in recent years due to their many applications especially in the fields of control $[1,2]$ and secure communi-

\footnotetext{
`Corresponding Author: Samir Bendoukha: Department of Electrical Engineering, College of Engineering, Yanbu, Taibah University, Saudi Arabia; Email: sbendoukha@taibahu.edu.sa

Adel Ouannas: Mathematics and Computer Science Department, Tebessa University,12062, Algeria

Amina-Aicha Khennaoui: Departement of Mathematics and Computer Sciences, University of Larbi Ben M'hidi, Oum El Bouaghi, Algeria

Giuseppe Grassi: Universita del Salento, Dipartimento Ingegneria Innovazione, 73100 Lecce, Italy

Xiong Wang: Institute for Advanced Study, Shenzhen University, Shenzhen, Guangdong 518060, China

Viet-Thanh Pham: Nonlinear Systems and Applications, Faculty of Electrical and Electronics Engineering, Ton Duc Thang University, Ho Chi Minh City, Vietnam
}

cations [3-6]. Several discrete-time chaotic systems have been proposed throughout the years including the well known 2-dimensional Hénon map [7], the Lozi system [8], the Zeraoulia-Sprott system [9], the generalized Hénon map [10] and the Baier-Klein system [11], and the discrete Rössler system [12]. These systems are all integer-order in the sense that the differences involved in the dynamical system's equations has an integer order. In recent years, researchers have picked up an interest in the fractional systems corresponding to the above mentioned integer-order systems. The basic idea is that fractional difference equations have an infinite memory, which both improves the flexibility of the dynamic model in representing physical phenomena as well as having the interesting property of infinite memory.

Continuous-time fractional calculus has been around for centuries. However its discrete counterpart is relatively new. Recent studies have attempted to formulate a framework for discrete-time fractional calculus whereby its stability and transformation theory is established [13-21]. Consensus has yet to be achieved as to the general notation and terminology. For that reason, we choose to adopt those of [22]. Over the last couple of years, substantial progress has been made in the subject of fractional discrete calculus. For instance, in [23], the authors considered a class of Caputo derivative-based generalized differential optimization problems. Interesting results were presented regarding the stability and discrete approximation of these problems. The stability of linear fractional difference systems with delay and impulse effects was treated in [24] using a novel Mittag-Leffler discrete function. Furthermore, a generalization of the Gronwall inequality was achieved in [25] and employed to establish the stability of discrete fractional delay systems.

The main aspect of dynamical chaotic systems that makes them so appealing is the seemingly unpredictable (random-like) nature of their trajectories in phase-space. Mathematically speaking, these are systems that have at least one positive Lyapunov exponent, which is defined

¿ Open Access. (c) 2019 A. Ouannas et al., published by De Gruyter. (cc) BY 4.0 License 
as the exponential growth in the difference between two trajectories starting from infinitesimally close initial states. At the end of the 1980s, chaos synchronization emerged whereby one chaotic or hyperchaotic (more than one positive Lyapunov exponent) can be controlled to follow the trajectory of another. This idea was revolutionary as it gave birth to chaos-based secure communications. In the simplest form of synchronization, the drive and response system states are controlled to become identical in infinite time. This is referred to as complete synchronization. In many applications, this is not required. Instead, what is required is that a certain function of the slave states synchronizes with a another function of the master states. This led to numerous synchronization types.

Numerous types of synchronization and control strategies have been proposed in the literature in relation to integer-order discrete-time systems including, for instance, [26-34]. As for fractional order discrete systems, the available literature is scarce and includes [35-39]. In our study, we are concerned with scalar matrix $\Theta-\Phi$ synchronization, which was first proposed in [40, 41] as a generalization of the matrix projective synchronization (MPS) scheme. The scheme was developed for continuous-time chaotic systems and numerical applications were shown. In [42], the authors extended the work to factional-order continuous systems. The importance of the $\Theta-\Phi$ lies in the fact that depending on the choice of the two scaling matrices, we may obtain different synchronization types including complete synchronization, anti-synchronization, matrix projective synchronization, and inverse matrix projective synchronization. The main contribution of this paper is the application of $\Theta-\Phi$ synchronization to fractionalorder chaotic maps with different dimensions. To the best of the authors' knowledge, most of the majority of results reported in the literature apply only to systems with identical dimensions, which makes the results presented herein both novel and interesting.

In the following section of this paper, we will define the notation and terminology to be used throughout the study. Section 3 details the proposed $\Theta-\Phi$ synchronization control laws with dimension $d=n$ and $d=m$. The convergence of the synchronization error is established by means of the stability theory of linear fractional-order discrete-time systems. In Section 4, we give two numerical examples that confirm the findings of our study. Finally, Section 5 summarizes the results of this paper.

\section{System Model}

Let us consider the master system given by

$$
{ }^{C} \Delta^{v} X(t)=A X(t+v-1)+f(X(t+v-1)),
$$

with $X(t)=\left(x_{1}(t), \ldots, x_{n}(t)\right)^{T}$ denoting its state vector, $A$ being an $n \times n$ real matrix, and $f: \mathbb{R}^{n} \rightarrow \mathbb{R}^{n}$ being a nonlinear function. Let us also consider as slave the $m$ component system

$$
{ }^{c} \Delta^{v} Y(t)=B Y(t+v-1)+g(Y(t+v-1))+U,
$$

where, again, $Y(t)=\left(y_{1}(t), \ldots, y_{m}(t)\right)^{T}$ are the states, $B \in \mathbb{R}^{m \times m}$, and $g: \mathbb{R}^{m} \rightarrow \mathbb{R}^{m}$ is a nonlinear function. The vector $U=\left(u_{i}\right)_{1 \leq i \leq m}$ is a controller used to drive the states of the slave system towards a specific trajectory to synchronize with the master. Finding a suitable closed form for this controller is the aim our paper.

Throughout this paper, we adopt the notation ${ }^{C} \Delta_{a}^{v} X(t)$ denoting the Caputo type delta difference of a function $X(t): \mathbb{N}_{a} \rightarrow \mathbb{R}$ with $\mathbb{N}_{a}=\{a, a+1, a+2, \ldots\}$ [16], which is of the form

$$
\begin{aligned}
{ }^{C} \Delta_{a}^{v} X(t) & =\Delta_{a}^{-(n-v)} \Delta^{n} X(t) \\
& =\frac{1}{\Gamma(n-v)} \sum_{s=a}^{t-(n-v)}(t-s-1)^{(n-v-1)} \Delta^{n} X(s),
\end{aligned}
$$

for $v / \in \mathbb{N}$ is the fractional order, $t \in \mathbb{N}_{a+n-v}$, and $n=\lceil\nu\rceil+1$. In (3), the $v$-th fractional sum of $\Delta_{s}^{n} X(t)$ is defined similar to [14] as

$$
\Delta_{a}^{-v} X(t)=\frac{1}{\Gamma(v)} \sum_{s=a}^{t-v}(t-s-1)^{(\nu-1)} X(s),
$$

with $v>0$. The term $t^{(v)}$ denotes the falling function defined in terms of the Gamma function $\Gamma$ as

$$
t^{(v)}=\frac{\Gamma(t+1)}{\Gamma(t+1-v)} .
$$

Normally, when we talk about synchronization, what comes to mind is trying to force the states of a slave dynamical system to coincide (synchronize) with those of a master. Traditionally, the slave and master were considered as the same system but with different initial conditions. For chaotic systems, this meant that the chaotic behavior of the slave is controlled through some parameter to synchronize to the master. Several forms of synchronization have been proposed throughout the years with different applications.

In our paper, we are concerned with what we call scaling matrix synchronization, which aims to show that 
through appropriate choice of $U$, there exist matrices $\Theta \in$ $\mathbb{R}^{d \times m}$ and $\Phi \in \mathbb{R}^{d \times n}$, with $d$ a certain dimension, such that the error

$$
e(t)=\Theta Y(t)-\Phi X(t),
$$

decays to zero as $t \longrightarrow+\infty$, i.e.

$$
\lim _{t \longrightarrow+\infty}\|e(t)\|=0 .
$$

If this is the case, then systems (1) and (2) are said to be $\Theta-\Phi$ synchronized in dimension $d$. It is easy to see that depending on our choice of the matrices $\Theta$ and $\Phi$, we may have several synchronization types:

- The pair $(\Theta, \Phi)=(I, I)$ yields complete synchronization as

$$
\lim _{t \longrightarrow+\infty}\|Y(t)-X(t)\|=0 .
$$

- The pair $(\Theta, \Phi)=(I,-I)$ yields antisynchronization as

$$
\lim _{t \longrightarrow+\infty}\|Y(t)+X(t)\|=0 .
$$

- The pair $(\Theta, \Phi)=(I, \Phi)$ yields matrix projective synchronization as

$$
\lim _{t \longrightarrow+\infty}\|Y(t)-\Phi X(t)\|=0 .
$$

- The pair $(\Theta, \Phi)=(\Theta, I)$ leads to inverse matrix projective synchronization as

$$
\lim _{t \longrightarrow+\infty}\|\Theta Y(t)-X(t)\|=0 .
$$

\section{Scaling Matrix Synchronization}

In this section, we present control law for the proposed $\Theta-\Phi$ synchronization scheme corresponding to two different cases of the synchronization dimension $d$, namely $d=m$ and $d=n$. In order to establish the convergence of the synchronization error in the two scenarios, we make use of the stability theory of linear fractional discrete systems, which can be summarized in the following theorem.

Theorem 1 [45] The zero equilibrium of the linear fractional-order discrete-time system

$$
{ }^{C} \Delta_{a}^{v} e(t)=\mathbf{M} e(t+v-1),
$$

where $e(t)=\left(e_{1}(t), \ldots, e_{n}(t)\right)^{T}, 0<v \leq 1, \mathbf{M} \in \mathbb{R}^{n \times n}$ and $\forall t \in \mathbb{N}_{a+1-v}$, is asymptotically stable if

$$
\lambda \in\left\{z \in \mathbb{C}:|z|<\left(2 \cos \frac{|\arg z|-\pi}{2-v}\right)^{v}\right.
$$

$$
\text { and } \left.|\arg z|>\frac{v \pi}{2}\right\} \text {, }
$$

for all the eigenvalues $\lambda$ of $\mathbf{M}$.

\subsection{Case 1: $d=m$}

Since in this case $d=m$, the scaling matrices are of the form $\Theta=\left(\Theta_{i j}\right)_{m \times m}$ and $\Phi=\left(\Phi_{i j}\right)_{m \times n}$. The $v$-Caputo fractional difference of the error system (6) can be derived as

$$
\begin{aligned}
{ }^{C} \Delta^{v} e(t) & =\Theta^{C} \Delta^{v} Y(t)-\Phi^{C} \Delta^{v} X(t) \\
& =\Theta B Y(t+v-1)+\Theta g(Y(t+v-1))+\Theta U \\
& -\Phi A X(t+v-1)+\Theta f(X(t+v-1)) .
\end{aligned}
$$

By defining a control matrix $C \in \mathbb{R}^{m \times m}$ and

$$
\begin{aligned}
R & =(C-B) e(t)+\Theta B Y(t)+\Theta g(Y(t))-\Phi A X(t) \\
& -\Theta f(X(t)),
\end{aligned}
$$

we can rewrite (10) in the form

$$
{ }^{C} \Delta^{v} e(t)=(B-C) e(t+v-1)+R+\Theta U,
$$

To achieve synchronization between systems (1) and (2), we assume that $\Theta$ is an invertible matrix and denote its inverse by $\Theta^{-1}$. The following theorem presents the control laws.

Theorem 2 The master-slave pair (1)-(2) is globally mdimensional $\Theta-\Phi$ synchronized by means of the control law

$$
U=-\Theta^{-1} \times R,
$$

subject to the control matrix $C$ being selected such that all the eigenvalues $\lambda$ of matrix $B-C$ satisfy

$$
-2^{v}<\lambda \leq 0 .
$$

Proof. Substituting (13) into (12), the fractional $\Theta-\Phi$ error system can be described as

$$
{ }^{C} \Delta^{v} e(t)=(B-C) e(t+v-1) .
$$

It is easy to see that subject to (13), all eigenvalues $\lambda$ of matrix $B-C$ satisfy $|\arg \lambda|=\pi>\frac{v \pi}{2}$ and $|\lambda|<2^{v}=$ $\left(2 \cos \frac{|\arg \lambda|-\pi}{2-\nu}\right)^{v}$. It, then, follows directly from Theorem 1 that the zero solution of the error system (15) is globally asymptotically stable and thus the master (1) and slave (2) are globally $m$-dimensional $\Theta-\Phi$ synchronized. 


\subsection{Case 2: $d=n$}

The second case we are going to consider is the one where the synchronization dimension $d=n$, where $n<m$, leading to the scaling matrices $\Theta=\left(\Theta_{i j}\right)_{n \times m}$ and $\Phi=\left(\Phi_{i j}\right)_{n \times n}$. Assuming a controllable matrix $L \in \mathbb{R}^{n \times n}$ and using the notation

$$
\begin{aligned}
T & =(L-A) e(t)+\Theta B Y(t)+\Theta g(Y(t))-\Phi A X(t) \\
& -\Theta f(X(t)),
\end{aligned}
$$

we may describe the error system in the form

$$
{ }^{C} \Delta^{v} e(t)=(A-L) e(t)+\Theta \times U+T .
$$

We assume that the matrix $\hat{\Theta}=\left(\Theta_{n \times n}\right)$ is invertible and denote its inverse by $\hat{\Theta}^{-1}$. The following theorem describes the proposed $n$-dimensional $\Theta-\Phi$ synchronization control laws .

Theorem 3 The master-slave pair (1)-(2) are globally $n$ dimensional $\Theta-\Phi$ synchronized if

$$
\hat{U}=\left(u_{1}, u_{2}, \ldots, u_{n}\right)^{T}=-\hat{\Theta}^{-1} \times T,
$$

and $u_{i}=0$ for $i=n+1, \ldots, m$, subject to the control matrix $L$ being selected such that all the eigenvalues of $A-L$ are situated between $-2^{v}$ and 0 .

Proof. Assuming that the components $u_{i}$ are equal to zero for $i=n+1, \ldots, m$, the product $\Theta \times U$ reduces to

$$
\Theta \times U=\hat{\Theta} \times \hat{U},
$$

where $\hat{U}=\left(u_{1}, u_{2}, \ldots, u_{n}\right)^{T}$ and $\hat{\Theta}=\left(\Theta_{n \times n}\right)$. Hence, the error system (17) becomes

$$
{ }^{C} \Delta^{v} e(t)=(A-L) e(t+v-1)+\hat{\Theta} \times \hat{U}+T .
$$

Substituting the proposed law (18) into (19) yields

$$
{ }^{C} \Delta^{v} e(t)=(A-L) e(t+v-1) .
$$

In much the same way as the proof of Theorem 2 and keeping in mind that the eigenvalues of $A-L$ satisfy the condition (14), the zero solution of error system (20) is globally asymptotically stable and, consequently, systems (1) and (2) are globally $n$-dimensional $\Theta-\Phi$ synchronized.

\section{Applications}

Let us now apply the findings of our study to a particular pair of Hénon-type discrete-time fractional-order maps.
We use the 2-dimensional system proposed in [43] as a fractional extension of the original integer-order system [7] to drive our synchronization. This master system is of the form

$$
{ }^{c} \Delta^{v} X(t)=A X(t+v-1)+f(X(t+v-1)),
$$

where

$$
A=\left(\begin{array}{cc}
-1 & 1 \\
b_{1} & -1
\end{array}\right)
$$

and

$$
f(X(t))=\left(\begin{array}{c}
-a_{1} x_{1}^{2}(t)+1 \\
0
\end{array}\right) .
$$

As for the slave system to be controlled, we use the fractional-order generalized chaotic 3-dimensional Hénon-like map [44], which is given by

$$
{ }^{c} \Delta^{v} Y(t)=B Y(t+v-1)+g(Y(t+v-1))+U,
$$

where

$$
B=\left(\begin{array}{ccc}
-1 & 0 & -b_{2} \\
1 & -1 & b_{2} \\
0 & 1 & -1
\end{array}\right),
$$

and

$$
g(Y(t))=\left(\begin{array}{c}
0 \\
0 \\
1-a_{2} y_{3}^{2}(t)
\end{array}\right)
$$

It is easy to show that these systems exhibit a chaotic behavior. Consider, for instance, the case where $\left(a_{1}, b_{1}\right)=$

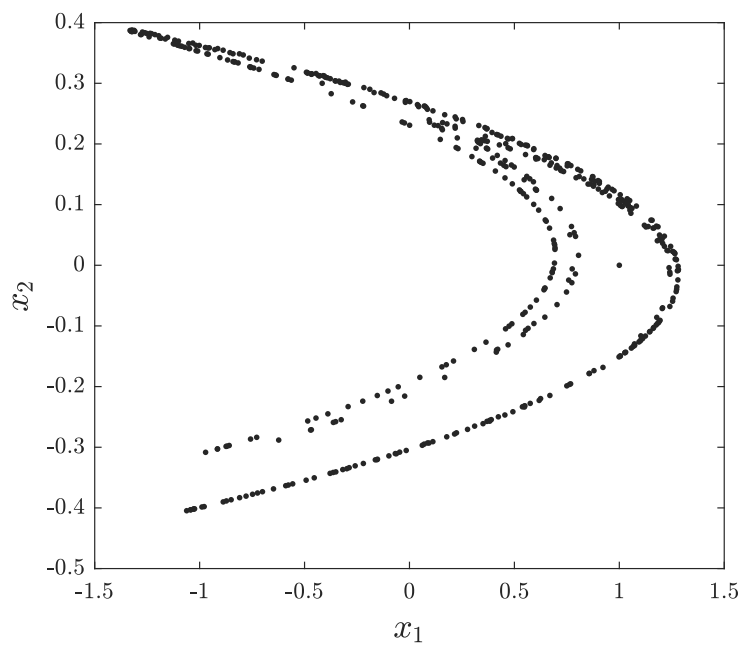

Figure 1: Phase space plot for the fractional Henon map with $\left(a_{1}, b_{1}\right)=(1.4,0.3), v=0.984$, and $x_{1}(0)=x_{2}(0)=0$. 

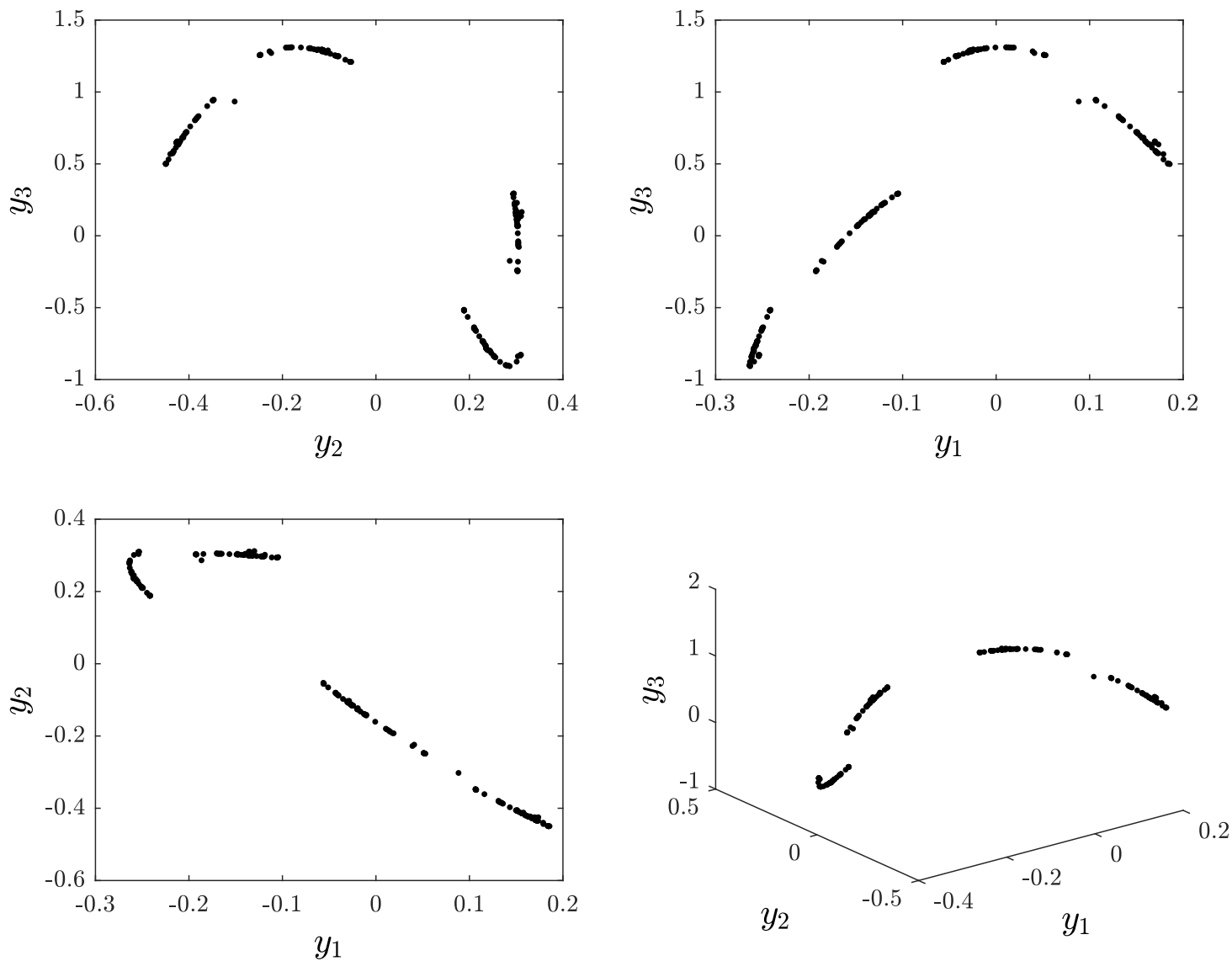

Figure 2: Phase portraits for the hyperchaotic Henon map with $\left(a_{2}, b_{2}\right)=(0.99,0.2), v=0.984$ and $\left(y_{1}, y_{2}, y_{3}\right)=(0.1,0.2,0.5)$.

$(1.4,0.3),\left(a_{2}, b_{2}\right)=(0.99,0.2)$, and $v=0.984$. For the uncontrolled slave, i.e. $U=(0,0,0)^{T}$, Figures 1 and 2 depict the chaotic trajectories of the master and slave, respectively.

We would like to put the results of Theorems 2 and 3 to the test and for that we consider two examples.

Example 1 Based on our approach described in Section 3.1, the $m$-dimensional $\Theta-\Phi$ error system is given by

$$
\begin{aligned}
\left(e_{1}(t), e_{2}(t), e_{3}(t)\right)^{T} & =\Theta\left(y_{1}(t), y_{2}(t), y_{3}(t)\right)^{T} \\
& -\Phi\left(x_{1}(t), x_{2}(t)\right)^{T}
\end{aligned}
$$

where

$$
\Theta=\left(\begin{array}{ccc}
1 & 0 & 0 \\
0 & 2 & 0 \\
-1 & 0 & 3
\end{array}\right) \text { and } \Phi=\left(\begin{array}{ll}
1 & 2 \\
0 & 1 \\
0 & 3
\end{array}\right) .
$$

Obviously, $\Theta$ is invertible and its inverse is given by

$$
\Theta^{-1}=\left(\begin{array}{ccc}
1 & 0 & 0 \\
0 & \frac{1}{2} & 0 \\
\frac{1}{3} & 0 & \frac{1}{3}
\end{array}\right)
$$

According to Theorem 2, there exists a matrix $C$ such that $(B-C)$ is negative-definite, which achieves $\Theta-\Phi$ synchronization. We choose, for instance,

$$
C=\left(\begin{array}{ccc}
0 & 0 & b_{2} \\
0 & 0 & -b_{2} \\
0 & 0 & 0
\end{array}\right) .
$$

It is clear that the eigenvalues of

$$
B-C=\left(\begin{array}{ccc}
-1 & 0 & 0 \\
1 & -1 & 0 \\
0 & 1 & -1
\end{array}\right)
$$

are equal to -1 and satisfy the condition of Theorem 1 . Control law (13) can be be achieved by means of matrix $R$ as defined in (11) and $\Theta^{-1}$ as in (29). According to Theorem 2 , once $U$ is defined as in (13) the master-slave pair becomes $m$-dimensional $\Theta-\Phi$ synchronized. This may be easily verified as the resulting error system is given by

$$
\left\{\begin{array}{l}
{ }^{C} \Delta^{v} e_{1}(t)=-e_{1}(t+v-1), \\
{ }^{C} \Delta^{v} e_{2}(t)=e_{1}(t+v-1)-e_{2}(t+v-1), \\
{ }^{C} \Delta^{v} e_{3}(t)=e_{2}(t+v-1)-e_{3}(t+v-1) .
\end{array}\right.
$$




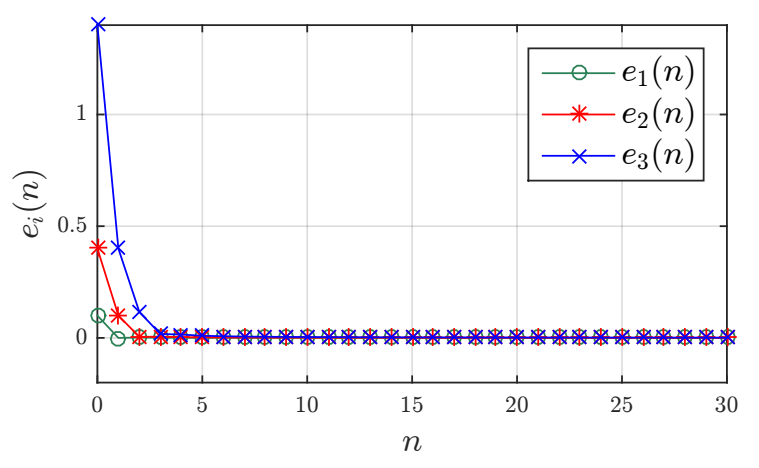

Figure 3: The evolution of errors over time for Example 1.

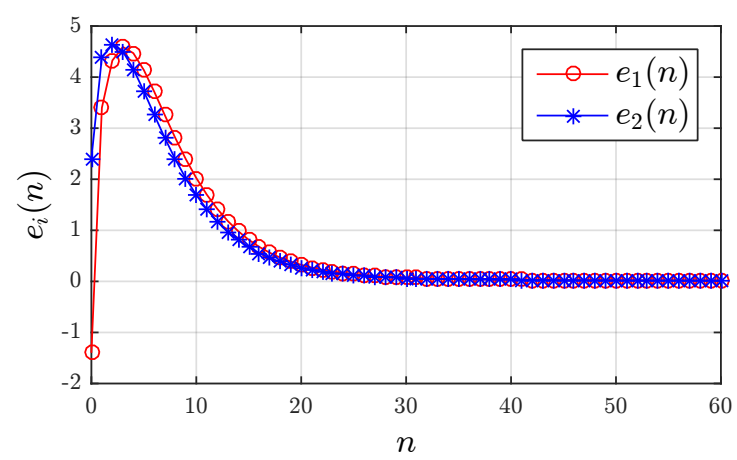

Figure 4: The evolution of errors over time for Example 2.

Given the initial values

$$
\left(\begin{array}{l}
e_{1}(0) \\
e_{2}(0) \\
e_{3}(0)
\end{array}\right)=\left(\begin{array}{l}
0.1 \\
0.4 \\
1.4
\end{array}\right),
$$

Figure 3 shows the convergence of the errors towards zero in sufficient time.

Example 2 Let us now move to the case considered in Section 3.2, the $n$-dimensional $\Theta-\Phi$ error system is of the form

$$
\begin{aligned}
\left(e_{1}(t), e_{2}(t)\right)^{T} & =\Theta\left(y_{1}(t), y_{2}(t), y_{3}(t)\right)^{T} \\
& -\Phi\left(x_{1}(t), x_{2}(t)\right)^{T}
\end{aligned}
$$

with

$$
\Theta=\left(\begin{array}{ccc}
1 & 0 & -1 \\
0 & 2 & 4
\end{array}\right) \text { and } \Phi=\left(\begin{array}{ll}
1 & 2 \\
1 & 3
\end{array}\right)
$$

Using the notation of Section 3.2, we have

$$
\hat{\Theta}=\left(\begin{array}{ll}
1 & 0 \\
0 & 2
\end{array}\right) \text { and } \hat{\Theta}^{-1}=\left(\begin{array}{cc}
1 & 0 \\
0 & \frac{1}{2}
\end{array}\right) .
$$

According to Theorem 3, there exists a control matrix $L$, which can be selected as

$$
L=\left(\begin{array}{cc}
0 & 0 \\
b_{1}+\frac{9}{16} & -\frac{3}{2}
\end{array}\right)
$$

leading to

$$
A-L=\left(\begin{array}{cc}
-1 & 1 \\
-\frac{9}{16} & \frac{1}{2}
\end{array}\right)
$$

which clearly satisfies the condition of Theorem 3 . Next, $T$ can be easily formed as in (16). Next, we may define the control law stated in (18) and append it with a zero at the end. Theorem 3 established that the master-slave pair (21)-(24) becomes $n$-dimensional inverse $\Theta-\Phi$ synchronized. To verify this, we use the error system

$$
\left\{\begin{array}{l}
{ }^{C} \Delta^{v} e_{1}(t)=-e_{1}(t+v-1)+e_{2}(t+v-1), \\
{ }^{C} \Delta^{v} e_{2}(t)=-\frac{9}{16} e_{1}(t+v-1)+\frac{1}{2} e_{2}(t+v-1) .
\end{array}\right.
$$

Figure 4 shows the time evolution of the errors given the initial values

$$
\left(\begin{array}{l}
e_{1}(0) \\
e_{2}(0)
\end{array}\right)=\left(\begin{array}{c}
-0.4 \\
2.4
\end{array}\right)
$$

\section{Summary}

In this paper, we dealt with the synchronization of fractional-order discrete-time chaotic systems. We considered the case of $\Theta-\Phi$ synchronization, which forces a linear function of the $n$ slave states represented as a matrix $\Phi \in \mathbb{R}^{d \times n}$ to converge towards a linear function of the $m$ master states represented by matrix $\Theta \in \mathbb{R}^{d \times m}$. This type of synchronization is powerful in that it encompasses numerous different synchronization schemes. We developed nonlinear control laws for two distinct cases of the dimension $d$, namely $d=m$ and $d=n$. The asymptotic stability and convergence of the synchronization errors were established by means of the stability theory of linear fractional discrete-time systems. Two numerical examples were presented using as master the fractional Hénon map and as slave the fractional 3-dimensional generalized Hénontype discrete map. Numerical results confirmed the existence of appropriate control matrices and the convergence of the synchronization error towards zero.

\section{References}

[1] Kocarev L., Szczepanski J., Amigo J.M., Tomovski I., Discrete Chaos-I: Theory, IEEE Trans. Circuits \& Systems, 2006, 53(6), 
1300-1309.

[2] Li C., Song Y., Wang F., Liang Z., Zhu B., Chaotic path planner of autonomous mobile robots based on the standard map for surveillance missions, Math. Prob. Eng., 2015, ID263964.

[3] Papadimitriou S., Bezerianosa A., Bountisb T., Pavlides G., Secure communication protocols with discrete nonlinear chaotic maps, J. Systems Architecture, 2001, 47(1), 61-72.

[4] Kwok H.S., Tang W.K.S., Man K.F., Online secure chatting system using discrete chaotic map, Int. J. Bifurcation Chaos, 2004, 14, 285.

[5] Banerjee S., Kurth J., Chaos and cryptography: a new dimension in secure communications, Eur. Phys. J. Special Topics, 2014 223, 1441-1445.

[6] Fataf N.A.A., Mukherjee S., Said M.R.M., Rauf U.F.A., Hina A.D., Banerjee S., Synchronization between two discrete chaotic systems for secure communications, Int. Conf. Comms. Elec. (ICCE), 2016, 477-481.

[7] Hénon M., A two-dimensional mapping with a strange attractor, Comms. in Math. Physics., 1976, 50(1), 69-77.

[8] Lozi R., Un atracteur étrange du type attracteur de Hénon, J. Physique, 1978, 39, 9-10.

[9] Zeraoulia E., Sprott J.C., The discrete hyperchaotic double scroll, Int. J. Bifurcation Chaos, 2009, 19, 1023-1027.

[10] Hitzl D.L., Zele F., An exploration of the Hénon quadratic map, Phys D Nonlinear Phenom, 1985, 14, 305-26.

[11] Baier G., Sahle S., Design of hyperchaotic flows, Phys. Rev. E, 1995, 51, 2712-2714.

[12] Yan Z.Y., Q-S (complete or anticipated) synchronization backstepping scheme in a class of discrete-time chaotic (hyperchaotic) systems : a symbolic-numeric computation approach, Chaos, 2006, 16, 013119-11.

[13] Atici F., Eloe P., A transform method in discrete fractional calculus, Int. J. Difference Equations, 2007, 2, 165-176.

[14] Atici F.M., Eloe P.W., Discrete fractional calculus with the nabla operator, Electron. J. Qual. Theory Differ. Equ. Spec. Ed. I, 2009, 3,1-12.

[15] Atici F.M., Senguel S., Modeling with fractional difference equations, J. Math. Anal. Appl., 2010, 369, 1-9.

[16] Abdeljawad T., On Riemann and Caputo fractional differences, Comput. Math. Appl., 2011, 62, 1602-1611.

[17] Holm M.T., The theory of discrete fractional calculus: development and application, PhD thesis, University of NebraskaLincoln, Lincoln, Nebraska, 2011.

[18] Mohan J., Deekshitulu G., Fractional order difference equations, Int. J. Differ. Equ., 2012, 1-11.

[19] Abdeljawad T., Baleanu D., Jarad F., Agarwal R.P., Fractional sums and differences with binomial coefficients, Discret. Dyn. Nat. Soc., 2013, 104173.

[20] Wu G.C., Baleanu D., Zeng S.D., Deng Z.G., Discrete fractional diffusion equation, Nonlin. Dyn., 2015, 80, 1-6.

[21] Baleanu D., Wu G.C., Bai Y., Chen F., Stability analysis of CaputoLike discrete fractional systems, Commun. Nonlinear Sci. Numer. Simul., 2017, 48, 520-530.

[22] Goodrich C., Peterson A.C., Discrete Fractional Calculus, Springer, 2015.

[23] Wu G.C., Baleanu D., Huang L.L., Novel Mittag-Leffler stability of linear fractional delay difference equations with impulse, Applied Mathematics Letters, 2018, 82, 71-78.

[24] Bai Y., Baleanu D., Wu G.C., Existence and discrete approximation for optimization problems governed by fractional differentia equations, Communications in Nonlinear Science and Numerical Simulation, 2018, 59, 338-348.

[25] Wu G.C., Baleanu D., Zeng S.D., Finite-time stability of discrete fractional delay systems: Gronwall inequality and stability criterion, Communications in Nonlinear Science and Numerical Simulation, 2018, 57, 299-308.

[26] Ouannas A., Azar A.T., Abu-Saris R., A new type of hybrid synchronization between arbitrary hyperchaotic maps, R. Int. J. Mach. Learn. \& Cyber., 2017, 8, 1887.

[27] Ouannas A., Grassi G., A new approach to study co-existence of some synchronization types between chaotic maps with different dimensions, Nonlinear Dynamics, 2016, 86(2), 1319-1328.

[28] Ouannas A., Odibat Z., Generalized synchronization of different dimensional chaotic dynamical systems in discrete-time, Nonlinear Dynamics, 2015, 81(1), 765-771.

[29] Ouannas A., A new generalized-type of synchronization for discrete chaotic dynamical systems, J. Comp. Nonlinear Dynamics, 2015, 10(6), 061019-5.

[30] Ouannas A., Grassi G., Inverse full state hybrid projective synchronization for vhaotic maps with different dimensions, Chiness Physics B, 2016, 25(9), 090503-6.

[31] Ouannas A., Grassi G., A new approach to study co-existence of some synchronization types between chaotic maps with different dimensions, Nonlinear Dynamics, 2016, 86(2), 1319-1328.

[32] Ouannas A., Odibat Z., Shawagfeh N., Universal chaos synchronization control laws for general quadratic discrete systems, Appl. Math. Model., 2017, 45, 636-641.

[33] Ouannas A., Grassi G., Karouma A., Ziar T., Wang X., Pham V.T., New Type of chaos synchronization in discrete-time systems: the F-M synchronization, Open Phys., 2018, 16, 174-182.

[34] Ouannas A., Grassi G., Karouma A., Ziar T., Wang X., Pham V.T., New type of chaos synchronization in discrete-time systems: the F-M synchronization, Open Physics, 2018, 16(1), 174-182.

[35] Wu G.C., Baleanu D., Chaos synchronization of the discrete fractional logistic map, Signal Process., 2014, 102, 96-99.

[36] Wu G.C., Baleanu D., Xie H., Chen F., Chaos synchronization of fractional chaotic maps based on the stability condition, Physica A, 2016, 460, 374-383.

[37] Liu Y., Chaotic synchronization between linearly coupled discrete fractional Hénon maps, Indian J. Phys., 2016, 90, 313-317.

[38] Kassim S., Hamiche H., Djennoune S., Bettayeb M., A novel secure image transmission scheme based on synchronization of fractional-order discrete-time hyperchaotic systems, Nonlinear Dyn., 2017, 88, 2473.

[39] Megherbi O., Hamiche H., Djennoune S., Bettayeb M., A new contribution for the impulsive synchronization of fractional-order discrete-time chaotic systems, Nonlinear Dyn, 2017, 90, 15191533.

[40] Ouannas A., Al-Sawalha M.M., A new approach to synchronize different dimensional chaotic maps using two scaling matrices, Nonlinear Dynamics and Systems Theory, 2015, 15(4), 400-408.

[41] Ouannas A., Al-sawalha M.M., Synchronization between different dimensional chaotic systems using two scaling matrices, Optik, 2016, 127, 959-963.

[42] Ouannas A., Al-sawalha M.M., Ziar T., Fractional chaos synchronization schemes for different dimensional systems with nonidentical fractional-orders via two scaling matrices, Optik, 2016, 127(20), 8410-8418.

[43] Hu T., Discrete Chaos in Fractional Henon Map, Appl. Math., 2014, $5,2243-2248$ 
[44] Shukla M.K., Sharma B.B., Investigation of chaos in fractional order generalized hyperchaotic Henon map, Int. J. Elec. Comm., 2017, 78, 265-273.
[45] Cermak J., Gyori I., Nechvatal L., On explicit stability condition for a linear fractional difference system, Fract. Calc. Appl. Anal., 2015, 18, 651-672. 